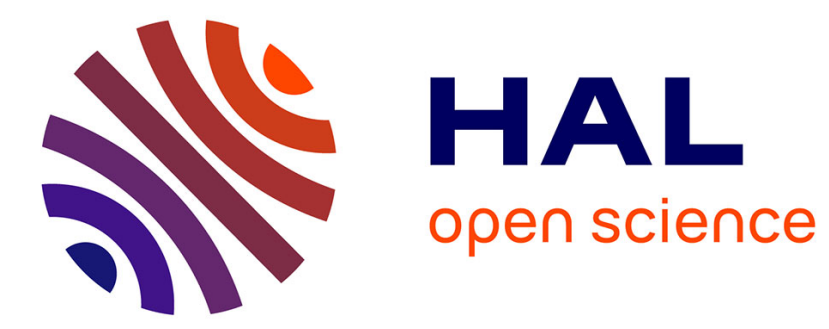

\title{
La bistabilité optique
}

S. Laval

\section{- To cite this version:}

S. Laval. La bistabilité optique. Revue de Physique Appliquée, 1984, 19 (2), pp.77-80. 10.1051/rphysap:0198400190207700 . jpa-00245176

\section{HAL Id: jpa-00245176 https://hal.science/jpa-00245176}

Submitted on 1 Jan 1984

HAL is a multi-disciplinary open access archive for the deposit and dissemination of scientific research documents, whether they are published or not. The documents may come from teaching and research institutions in France or abroad, or from public or private research centers.
L'archive ouverte pluridisciplinaire HAL, est destinée au dépôt et à la diffusion de documents scientifiques de niveau recherche, publiés ou non, émanant des établissements d'enseignement et de recherche français ou étrangers, des laboratoires publics ou privés. 


\title{
La bistabilité optique
}

\author{
S. Laval \\ Institut d'Electronique Fondamentale, Université Paris XI, Bâtiment 220, 91405 Orsay Cedex, France
}

(Reçu le 3 juin 1983, accepté le 19 octobre 1983)

\begin{abstract}
Résumé. - Les dispositifs bistables sont les éléments de base pour réaliser en optique des fonctions logiques. Les
\end{abstract} différents types de systèmes sont brièvement décrits, ainsi que quelques applications.

Abstract. - Bistable devices must be developed to obtain logic functions in optics as in electronics. The various kinds of systems are briefly described, and some applications are given.

\section{Introduction.}

Depuis l'apparition des lasers, on a cherché constamment à tirer le meilleur parti des fréquences élevées des ondes optiques, que ce soit pour la transmission ou pour le traitement de l'information. Le développement des télécommunications optiques en est un exemple bien connu. En ce qui concerne le traitement du signal, les circuits logiques sont largement utilisés en électronique. La possibilité de réaliser des fonctions logiques à partir de signaux optiques a été entrevue vers 1970 , où, pour la première fois, un composant présentant au moins deux états de transmission de lumière a été proposé $[1,2]$. Il a fallu attendre 1975 pour que la preuve expérimentale soit apportée et que la bistabilité optique soit effectivement observée [3]. Depuis, les études sur ce sujet se sont rapidement multipliées.

Compte tenu des applications potentielles, de nombreux efforts ont porté sur les possibilités d'intégration de tels dispositifs et sur l'amélioration de leurs performances, en particulier quant aux temps de commutation qu'ils permettent d'obtenir.

\section{Principe de fonctionnement d'un dispositif bistable optique.}

Un dispositif bistable est caractérisé par l'existence, dans une certaine zone de fonctionnement, des deux états stables, c'est-à-dire de deux niveaux de sortie possibles pour une intensité donnée à l'entrée. La commutation de l'un à l'autre de ces niveaux peut se faire par l'intermédiaire d'un changement temporaire de l'intensité incidente. Si l'on représente les variations du signal de sortie en fonction du signal d'entrée, la bistabilité se caractérise par un cycle d'hystérésis (Fig. 1). Pour l'obtenir, il faut que le système soit non linéaire, et qu'il existe une réaction du signal de sortie sur le signal d'entrée.

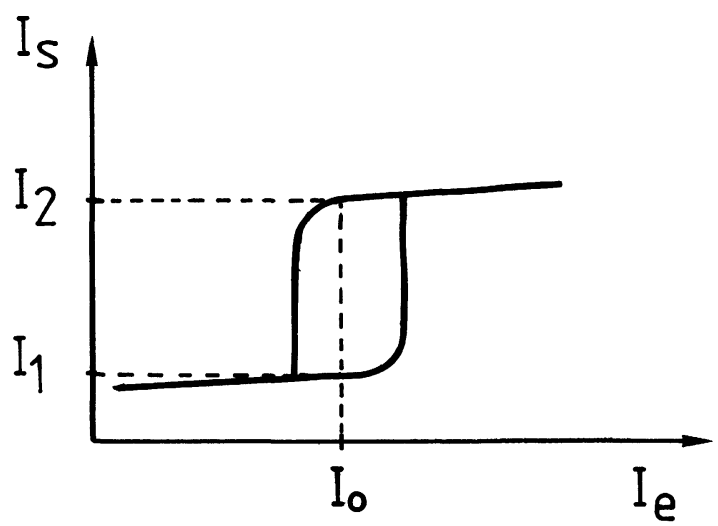

Fig. 1. - Caractéristique du signal de sortie en fonction du signal d'entrée d'un système bistable.

[Output versus input signal for a bistable device.]

Les premières mises en évidence expérimentales de la bistabilité optique $[3,5]$ ont été faites sur un dispositif constitué simplement d'un matériau non linéaire, dont l'indice de réfraction varie avec l'intensité lumineuse, placé dans un résonateur Pérot-Fabry, dans lequel la lumière partiellement réfléchie par le miroir de sortie réagit sur le système (Fig. 2).

Rappelons que la transmission d'une cavité Pérot- 


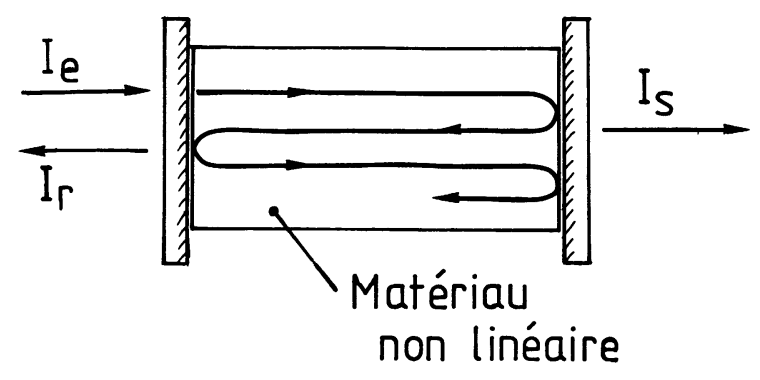

Fig. 2. - Dispositif bistable intrinsèque constitué d'une cavité Pérot-Fabry remplie d'un matériau non linéaire.

[Optical bistable device consisting of a Fabry-Perot resonator containing a non linear material.]

Fabry dont chaque miroir présente un coefficient de réflexion $R$ est donnée par l'expression [6]

$$
T=\frac{I_{\mathrm{s}}}{I_{\mathrm{e}}}=\frac{1}{1+\frac{4 R}{(1-R)^{2}} \sin ^{2} \frac{\phi}{2}}
$$

où $\phi$ représente le retard de phase de la lumière de longueur d'onde $\lambda$ ayant parcouru un aller-retour dans la cavité de longueur $l$ :

$$
\phi=\frac{2 \pi n l}{\lambda}
$$

$n$ étant l'indice de réfraction à l'intérieur de la cavité.

La transmission est maximale lorsque les ondes se retrouvent en phase après un aller-retour, donnant lieu à des interférences constructives, c'est-à-dire lorsque le chemin optique $n l$ dans la cavité est un multiple entier de la longueur d'onde. Cette condition de résonance fait que l'intensité de la lumière dans la cavité dépend de la longueur du chemin optique. Lorsque cette dernière varie elle-même avec l'intensité lumineuse, les conditions nécessaires à l'existence de la bistabilité sont remplies.

Supposons que l'indice de réfraction varie linéairement avec la puissance optique, ce qui est le cas par exemple par effet Kerr, l'indice peut s'écrire :

$$
n=n_{0}+n_{2} I_{\mathrm{int}}
$$

où $n_{2}$ est un coefficient qui caractérise la non-linéarité du milieu intracavité, et $I_{\text {int }}$ la puissance lumineuse dans le résonateur, qui est proportionnelle à l'intensité transmise $I_{s}$.

La phase $\Phi$ s'exprime alors sous la forme

$$
\Phi=\Phi_{0}+K I_{\mathrm{s}}
$$

qui, joint à la relation 1 permet de déterminer les variations de la transmission du dispositif bistable en fonction du niveau d'entrée $I_{\mathrm{e}}$. Les solutions peuvent être trouvées par une résolution graphique (Fig. 3), à l'intersection de la courbe donnant classiquement la transmission d'un Pérot-Fabry (Eq. 1) et de droites

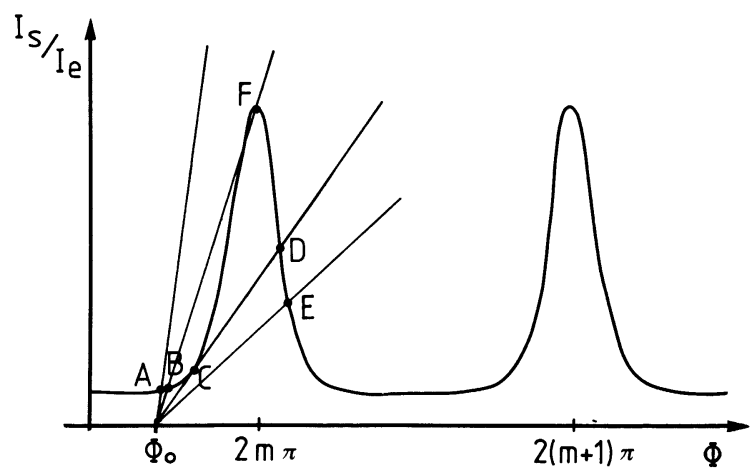

Fig. 3. - Résolution graphique permettant de déterminer les solutions correspondant au fonctionnement bistable.

[Graphical resolution for bistable solutions.]

dont la pente est inversement proportionnelle à $I_{\mathrm{e}}$ (Eq. 3). Lorsque l'intensité incidente est faible, $\Phi_{0}$ ne correspondant pas à une résonance de la cavité, la transmission est faible (point A). Pour des valeurs croissantes de l'intensité, la transmission varie légèrement jusqu'au point $C$ à partir duquel il n'existe plus de solution par continuité, et le niveau de sortie passe alors à un état haut, correspondant au point $\mathrm{D}$. Pour des intensités décroissantes, l'état de forte transmission est conservé jusqu'au point $F$, avant que le système ne repasse dans un état de faible transmission (point B). On voit ainsi apparaître le cycle caractéristique de la bistabilité, dont la largeur dépend du point de fonctionnement défini par la valeur de $\phi_{0}$.

\section{Dispositifs intrinsèques et hybrides.}

La difficulté à trouver des matériaux de bonne qualité optique et présentant des non-linéarités importantes a conduit à développer des dispositifs hybrides, dans lesquels un détecteur convertit le signal optique de sortie en un signal électrique, qui peut être amplifié, et est appliqué sur un cristal électro-optique pour créer la non-linéarité [7]. Ceci permet d'obtenir des systèmes fonctionnant à des puissances lumineuses beaucoup plus faibles que dans le cas des dispositifs intrinsèques, comme celui décrit ci-dessus, car les non-linéarités induites sont très largement supérieures à celles obtenues à partir de l'onde lumineuse elle-même.

Une boucle de contre-réaction électronique introduit par ailleurs un certain nombre de paramètres supplémentaires qui ont souvent été mis à profit pour étudier le fonctionnement du dispositif, en particulier en ce qui concerne le régime transitoire. Par contre, elle limite le temps de réponse du système par ses propres constantes de temps.

L'effet électro-optique est depuis longtemps utilisé en optique intégrée dans la réalisation de coupleurs ou de modulateurs. L'adjonction d'un détecteur fournissant un signal de contre réaction permet d'obtenir la bistabilité [8], et de nombreux efforts ont été faits en ce sens, afin de miniaturiser les systèmes. Les 
structures de base généralement utilisées sont celles des coupleurs directionnels [9] ou d'interféromètres à deux bras de type Mach-Zehnder [10]. La concentration de l'énergie lumineuse dans les guides d'onde optiques abaisse le seuil de puissance nécessaire. De plus, on peut par ces techniques envisager la possibilité de réaliser plusieurs dispositifs sur le même substrat, et de progresser ainsi vers la définition de systèmes intégrés plus complexes [11].

Une autre voie vers la miniaturisation consiste à mettre en jeu les non-linéarités importantes existant au voisinage du seuil d'absorption dans un semiconducteur [12] comme InSb ou GaAs. La cavité Fabry-Pérot est alors formée par les faces du cristal et le dispositif est d'encombrement très réduit. Les inconvénients résident dans le domaine spectral très limité dans lequel fonctionnent de tels dispositifs, fixé par le choix du matériau, et dans l'utilisation de basses températures, mais des résultats très prometteurs ont été obtenus sur des structures à puits quantiques [13] à la température ambiante.

Si la réduction des dimensions est un point important du point de vue des applications pratiques des dispositifs bistables optiques, il est également essentiel, si l'on veut réaliser des dispositifs compétitifs par rapport à l'électronique, de réduire au maximum les temps de commutation. Plusieurs facteurs réduisent la rapidité : les constantes de temps du circuit électronique dans le cas des systèmes hybrides, le temps de remplissage de la cavité Fabry-Pérot pour les systèmes classiques, intrinsèques ou hybrides tels qu'on les a décrits, et enfin les temps de relaxation propres au matériau non-linéaire. Des systèmes intrinsèques, et sans cavité résonnante, ont été réalisés pour s'affranchir des deux premiers facteurs, et atteindre ainsi des vitesses de commutation de l'ordre de la picoseconde avec des matériaux présentant un effet Kerr d'origine électronique. Le premier de ces systèmes utilise la réflexion totale de la lumière sur un matériau non linéaire [14] $\left(\mathrm{CS}_{2}\right)$. Par l'intermédiaire de l'onde évanescente les variations d'indice induites provoquent une modification de l'angle critique telle que l'on peut passer d'un état de réflexion totale à un état de transmission et réciproquement. On peut également utiliser

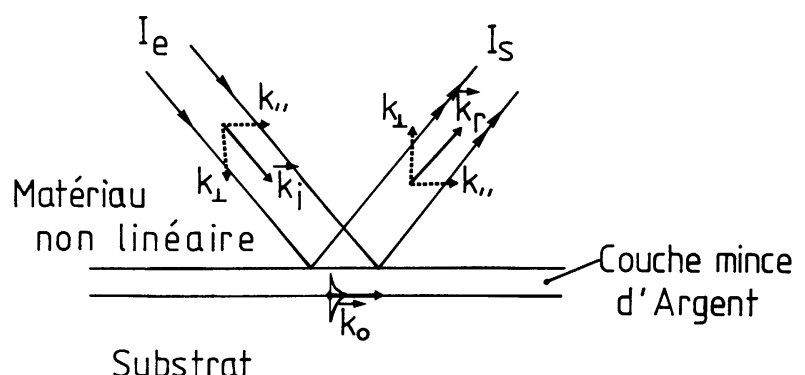

Fig. 4. - Schéma d'un dispositif bistable intrinsèque basé sur l'excitation résonnante d'une onde de surface.

[Intrinsic bistable optical device by resonant excitation of a surface wave.]

REVUE DE PHYSIQUe APPLIQUÉE. - T. 19, № 2, FÉVRIER 1984 les variations de réflectivité associées à l'excitation résonnante d'une onde de surface par réflexion totale frustrée [15]. Les faisceaux incidents et réfléchis se propageant au travers d'un milieu non-linéaire (Fig. 4), en modifient l'indice de réfraction, et donc l'accord par rapport au vecteur d'onde de l'onde de surface que l'on veut exciter. On passe ainsi d'un état de réflectivité très faible, quand on est proche de la résonance, à un état de réflectivité élevée, dans lequel le faisceau réfléchi contribue à la modification de l'indice, introduisant ainsi le processus de contre-réaction. Nous avons mis en évidence la bistabilité dans un tel système en excitant une onde de plasmons de surface, à une interface argent-silice, au travers de sulfure de carbone et en mettant en jeu dans cette étude de faisabilité des variations d'indice induites thermiquement [16]. Le cycle d'hystérésis apparaît à partir d'un certain angle d'incidence, et s'élargit quand cet angle augmente (Fig. 5). Simultanément, la bistabilité a lieu pour des puissances incidentes plus élevées.

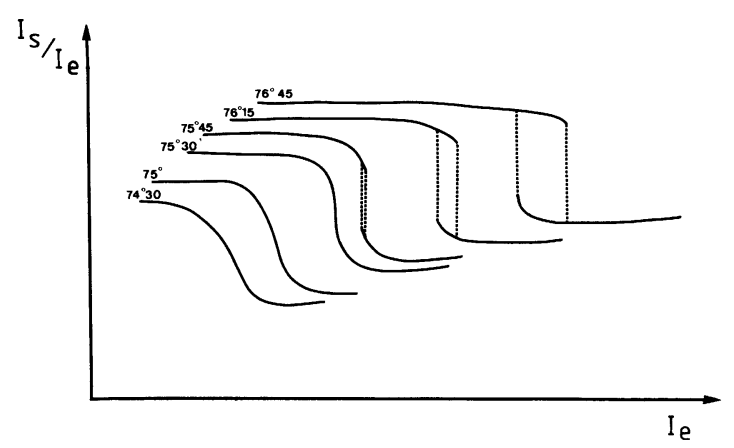

Fig. 5. - Variations de la réflectivité en fonction de la puissance d'entrée pour différents angles d'incidence sur le dispositif représenté figure 4 .

[Reflexion coefficient versus input power for various incidence angles on the device represented in figure 4.]

\section{Applications.}

Les plus optimistes vont jusqu'à prévoir des ordinateurs optiques [17]. Il est certain qu'à partir de caractéristiques du type de celles représentées sur la figure 5, de nombreuses applications peuvent être envisagées [7]. Une caractéristique sans hystérésis peut être utilisée pour réaliser des fonctions de limiteur optique. et de mise en forme d'impulsions. On peut réaliser des opérations logiques du type des portes «ET » pour lesquels deux signaux (inférieurs au seuil) sont nécessaires à l'entrée pour obtenir l'état haut à la sortie, ou du type des portes «OU » dans lequel un signal sur l'une quelconque des 2 entrées est suffisant pour que le niveau haut soit obtenu à la sortie. Un tel dispositif peut également être utilisé en transistor optique, un faible signal de commande provoquant la commutation d'un état à l'autre lorsque le système est prééclairé.

Lorsque le cycle d'hystérésis existe, le dispositif bistable a les fonctions d'une mémoire puisqu'il reste 
dans l'un des deux états possibles si aucune perturbation ne lui est appliquée.

On peut noter que les dispositifs optiques sont bien adaptés à un traitement de l'information en parallèle, en envoyant plusieurs faisceaux en des points différents du matériau non linéaire, et ceci peut ouvrir des voies nouvelles du point de vue de la conception de circuits complexes.
Il existe donc une large gamme d'applications, mais il subsiste un certain nombre de problèmes à résoudre, qui en conditionneront le développement effectif, en particulier en ce qui concerne la nécessité de disposer de matériaux optiques transparents et de bonne qualité dont les coefficients non linéaires d'origine électroniques soient importants.

\section{Bibliographie}

[1] Szoke, A., Daneau, V., Goldhar, J. et Kurnit, N. A., Appl. Phys. Lett. 15 (1969) 376.

[2] Austin, J. W. et Deshazer, L. G., J. Opt. Soc. Amer. 61 (1971) 650.

[3] McCall, S. L., Gibbs, H. M. et Venkatesan, T. N. C., J. Opt. Soc. Am. 65 (1975) 1184.

[4] Gibis, M. M., McCall, S. L. et Venkatesan, T. N. C., Phys. Rev. Lett. 36 (1976) 1135.

[5] Venkatesan, T. N. C. et McCall, S. L., Appl. Phys. Lett. 30 (1977) 282.

[6] Born, M. et Wolf, E., Principle of Optics (Pergamon Press) 1964.

[7] Smith, P. W., Turner, E. H. et Maloney, P. J., IEEE J. QE 14 (1978) 207.

[8] Smith, P. W., Kaminow, I. P., Maloney, P. J. et Stulz, L. W., Appl. Phys. Lett. 33 (1978) 24.

[9] Carenco, A. et Menigaud, L., Appl. Phys. Lett. 37 (1980) 880.
[10] Schnapper, A., Papuchon, M. et Puech, C., Optics Communs. 29 (1979) 364.

[11] Ito, H., Ogawa, Y. et Inaba, H., IEEE J. $Q E 17$ (1981) 325.

[12] Miller, D. A. B., Smith, S. D. et Seaton, C. T., IEEE J. QE 17 (1981) 312.

[13] Miller, D. A. B., Chemla, D. S., Eilenberger, D. J., Smith, P. W., Gossard, A. C. et Tsang, W. T., Appl. Phys. Lett. 41 (1982) 679.

[14] Smith, P. W., Hermann, J. P., Tomlison, W. J. et Maloney, IEEE J. QE 17 (1981) 340.

[15] Martinot, P., Koster, A., Laval, S. et Carvalho, W., Appl. Phys. B 29 (1982) 172.

[16] Martinot, P., Thèse 3e cycle, Orsay, juin 1983.

[17] Abraham, E., Seaton, C. et Smith, D., Pour la Science (1983) 85. 